\title{
L'Amour cyclique Proustien
}

Mary Gayle Pifer

A 1a Recherche du Temps perdu, 1'oeuvre de Marcel proust a été souvent comparée à une composition musicale qui présenterait un entrelacement continu d'un certain nombre de leitmotivs tels que rêves, temps, habitude, mémoire, art, amour, mort, société et amitié. Proust ne permet à aucun de ces thèmes d'atteindre une condition de résolution complète avant d'avoir été interrompu par un des autres thèmes présentés dans ce roman et mêlés à lui. Comme il y a rappel des mêmes mélodies dans une oeuvre dramatique musicale, dans I'oeuvre de Proust, chaque thème est présenté dans des variations et tous les thèmes sont intervertis et leur intensité augmentée. Le modèle établi par la conduite amoureuse de Swann démontre le caractère de 1 'amour dont Proust dit qu'il suit toujours des "lois immuables et naturelles."1 Ainsi les passions de l'oeuvre suivent un modèle cyclique identique. Cependant, chaque fois que le thème de 1 'amour apparaît, certains aspects de ce modèle cyclique et des "lois immuables" qui en résultent sont intensifiés comme les variations d'une même mélodie. Par exemple, le narrateur compare directement sa jalousie enfantine à celle de Swann et ce dernier annonce le thème qui fera le sujet de la Prisonnière.

Parmi les cinq passions principales de $\underline{\mathrm{A}}$ la $\mathrm{re}-$ cherche du temps perdu (Swann-Odette, Marcel-Gilberte, Marcel-Albertine, Saint-Loup-Rache1, Charlus-Morel), 1'épisode d'Un Amour de Swann précise très clairement les lois et les structures cycliques de 1'amour. En général le modèle cyclique de 1'amour, tel qu'il est 
établi dans Un Amour de Swann présente: (1) une période d'indifférence, composée d'ennui et d'une disposition à $I^{\prime}$ amour; (2) une période de souffrance, basée sur les qualités subjectives de 1 'amour qui conduisent à la jalousie (une période qui se termine en un sentiment de culpabilité difficile à analyser et produit par un désir de revanche); (3) un retour à l'indifférence, qui est en partie uen aliénation due à une libération de la souffrance et en partie également une expansion esthétique de 1 'âme. Les interruptions dans ce modèle cyclique de 1 'amour représentent 1 'impulsion qui prépare I'étape suivante du cycle. (Se reporter au bref diagramme suivant.) Chaque étape dépend de la phrase précédente du modèle.

INDIFFERENCE ET ENNUI

Un Amour de Swann n'est pas une oeuvre romanesque où 1'amour naît d'un coup de foudre. Odette lui parâ̂t posséder "un genre de beauté qui lui est indifférent" (p. 195). Au début de leur amour Odette poursuit Swann, renversant par ses actions le rôle traditionnel de son sexe. On peut expliquer cette attitude parce qu'elle est dans une position sociale bien moins assurée que celle de Swann. Celui-ci prend 1'habitude de voir Odette régulièrement par ennui ou par inertie.

\section{INDIFFERENCE ET INCLINAISON}

Il faut remarquer que les individus implịqués dans ce cycle présentent toujours des traits de caractère personnels opposés de façon particulière. Proust, de façon cohérente, met en contact un amant intelligent, cultivé et riche, ayant la capacité et le besoin d'aimer, avec un être aimé sans éducation et pauvre, n'ayant aucune inclinaison à aimer. L'être aimé profite de l'amant sans être entraîné lui-même à aimer tandis que celui-ci souffre pendant toute la durée de leur amour. Si les individus assortis de cette façon avaient des traits de caractère personnels différents, le cycle n'aurait pas à se dérouler nécessairement comme prévu. Cependant 1 'hypothèse cachée dans le concept de $1^{\prime}$ amour proustien est que c'est précise- 
ment et exclusivement des individus qui ont ces caractères opposés qui s'attirent mutuellement et éprouvent un amour à sens unique. En fait, $c^{\prime}$ est le problème de la mise en esclavage d'un homme sensible, intellectuellement supérieur par une femme inférieure et stupide, ou plutôt, l'asservissement d'un homme à ses propres émotions.

Odette dont le rôle à l'origine avait été de pourchasser Swann, lui abandonne peu à peu le rôle actif. Progressivement 1 'idée que peut-être, Odette pourrait se lasser de sa compagnie le préoccupe. Mais à l'instant même où il se croit délivré sincèrement d'Odette, "1a petite phrase" de la sonate de Vinteuil qu'il entendait sans cesse avec elle, tisse son sortilège. Cette mélodie devient "1'air national" (p. 218) de ses rapports avec Odette.

En entendant cette phrase musicale, l'état d'esprit de Swann est enclin à $I^{\prime}$ amour. Comme lorsqu'il s'agit d'hypnose, 1'amour romanesque est un état d'âme qui, au départ, exige un consentement. Une fois que $I^{\prime}$ on est tombé en extase, la possibilité de reculer est perdue. Swann a atteint un âge dangereux et il se demande ce qu'il éprouverait à vivre uniquement pour 1 'amour comme le font les personnages de livres. La musique semblait lui parler d'un monde privilégié, d'un royaume supérieur de vérités éternelles, d'un rajeunissement possible, délivré de son inertie spirituelle. Il veut s'enrichir au contact d'un autre être, mais cette faim sera nourrie par une souffrance presque diabolique.

Devenu amoureux d'Odette, Swann va lui rendre visite dans son appartement. En actrice pleine d'affectation, elle s'est entourée de chrysanthèmes et d'orchidées précisément parce que ces fleurs manquent de "naturel". Conscient peut-être des imperfections de la jeune femme, donc de sa propre faiblesse, Swann cherche à justifier sa passion par des motifs d'ordre esthétique. Il commence par discerner une profonde ressemblance entre Odette et une peinture de Boticelli. A ce moment-là, se produit une des plus importantes transmutations des sentiments de Swann envers son amie. 
L'esclavage de Swann croît jusqu'au moment où Odette quitte la réunion quotidienne des Verdurin avant son arrivée. Le choc de cette absence inattendue déclenche le mécanisme de 1 'amour. Le développement du désir d'aimer comme de toutes les habitudes diverses dont il a permis la croissance, a rétréci le champ de son indifférence. Un incident inattendu détermine la transformation de sa quasi-indifférence à l'état d'amour subjectif et impuissant. A cause de I'absence inattendue d'Odette, Swann souffre et soudain "sa vie lui paraissait plus intéressante" ( $p$. 229). Son état d'âme se réduit à une oscillation entre l'ennui et la douleur. Aussitôt qu'un des éléments disparaît, $1^{\prime}$ autre prend sa place. A l'instant même où $1^{\prime}$ homme croit atteindre le bonheur, celui-ci s'évanouit.

Donc le bonheur en amour consiste en cette courte pause au moment où 1 a souffrance a cessé et où $I^{\prime}$ ennui n'a pas encore eu le temps de naître. Proust présente la genèse de I'amour lorsqu'il décrit Swann chérchant avec désespoir Odette.

\section{DESESPOIR ET DESILLUSION - AMOUR SUBJECTIF}

De combien d'erreurs s'accompagne 1'amour! Son imagination crée et façonne l'être cher, qui devient inconsistant. Dès que cet être se montre à ses yeux de chair il disparaît au regard de son esprit car 1 'amour est moins Odette elle-même que le désir de Swann. En apparence, 1'amour peut paraitre agir de l'extérieur quand réellement il est influencé par une force intérieure.

Proust s'efforce d'utiliser une abondance d'images plutôt qu'un vocabulaire abstrait afin d'obtenir une impression particulière et d'établir des rapports entre 1'art et 1'amour. Quand Proust veut créer des comparaisons très efficaces entre ce qu'il appelle le monde réel et le monde imaginaire, il se sert d'une

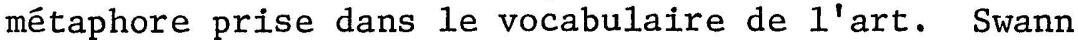
n'est pas le seul personnage à utiliser l'esthétique pour créer une image illusoire de 1 'être aimé. 
Charlus compare son bien-aimé, Morel, à diverses oeuvres d'art. Le narrateur fait de même avec son amie Albertine.

Le thème de la nature subjective, affective de 1 l'amour existe dès les premières pages du roman. Le narrateur décrit son sommeil troublé et ses rêves de plaisir donné par une femme imaginaire. On peut dire qu'Odette, Albertine, Gilberte, Rachel et Morel, qui représentent les cinq passions principales, sont des créations de l'esprit de leurs amants au même titre que la femme rêvée était la création de l'esprit du rêveur.

Soudain Swann se trouve face à face avec Odette. Tandis qu'il se penche au-dessus d'elle pour étingler une fleur, un catleya, ils s'étreignent et échangent un baiser pour la première fois. Le visage d'Odette cesse alors d'exister pour Swann, I1 y substitue l'image mentale créée par son désir et le catleya devient le symbole de la possession physique. Ceci est accompagné d'une déclaration cynique sur la monotonie de la possession des femmes. Pour Proust le catleya est une représentation de la stérilité de I'amour, un acte d'autofécondation, puisque la femme aimée est une création subjective, une projection mentale créée par l'amant.

Swann est complètement désarmé par 1 'expression qu' il descerne parfois sur le visage d'Odette et qui, d'une certaine manière, lui rappelle sa mère. Proust, comme Freud, voit une analogie entre le modèle d'un amour individuel et 1 a nature des relations qu'il avait avec ses parents. Swann est faussement rassuré quand la perfide Odette a une expression qui lui rappelle sa propre mère. Le cas de Mlle Vinteuil est peut-être le plus poignant exemple de cette analogie car elle ne peut éprouver de plaisir que quand son amie crache sur $l^{\prime}$ image de son père mort. Le narrateur compare les baisers de sa mère quand il était enfant avec les baisers de son amie. Sa jalousie, son angoisse dans $1^{\prime}$ attente du baiser de sa mère font penser au complexe d'Oedipe et à un amour de l'impossible. Le narrateur, obsédé par son image familiale et par un désir de faire rétribution de cette culpabilité sans 
nom, cherche inconsciemment un amour qui lui représente toujours l'impossible et la souffrance. Il faut, donc, que la psychologie des êtres aimés, hétérosexuels ou homosexuels, soit incomplète pour rendre aux amants la souffrance nécessaire, soit pour une expiation, soit pour une quête d'une vérité éternelle. En effet, le narrateur cherche cette dernière dans la passion et trouve que 1'amour est stêrile. Il se rend compte qu'un homme subit les lois de son passé et de son image familiale et qu'il doit chercher, réparer son "temps perdu" pour découvrir le vrai chemin vers une vérité éternelle.

\section{DESESPOIR ET DESILLUSION - JALOUSIE (OMBRE DE L'AMOUR)}

De plus en plus entraîné par son amour pour Odette, au point de lui donner de 1 'argent tous les mois, il refuse de croire les gens qui lui disaient: "C'est pour ta fortune qu'elle t'aime." (p. 267) Il faut noter que 1'amour n'est jamais inspiré par une prostituée qui se déclare telle ouvertement. En effet, il doit $y$ avoir toujours un risque d'impossibilité pour savourer la possession de la personne aimée. Mais comme, d'autre part, les femmes vertueuses sont impuissantes à inspirer l'amour, seules les femmes dont la vertu est douteuse intéressent parce qu'elles semblent capables de succomber à l'attrait de l'argent ou d'une position sociale.

A ce point, Swann éprouve sa première attaque de jalousie. Il imagine qu'Odette $1^{\prime}$ 'a renvoyé de bonne heure afin de recevoir quelqu'un d'autre. Proust excelle à dépeindre la jalousie, cette "ombre de 1 'amour" ( $p .276$ ), comme une phase spéciale de l'expérience humaine. L'amour réciproque semble impossible à ses yeux, car la cristallisation peut seulement s'effectuer après que le personnage ait souffert du doute et de la jalousie. Nous désirons ce que nous ne pouvons pas posséder et l'amour, une fois obtenu, cesse d'être désirable. C'est la raison pour laquelle le bonheur en amour est par nature impossible puisqu'il exige des rapports spirituels irréalisables. L'amour 
comme le désir de la possession de ce bonheur est détruit par cette possession elle-même. La fonction créatrice de la jalousie est l'impuissance de supporter l'absence de l'être aimé et l'impuissance de supporter l'esprit de cette personne. La jalousie transforme I'amour complètement mais en même temps, sans elle, I'amour ne se renouvellerait pas et perdrait de son intensité. Toute nouvelle souffrance qu'on nous inflige, augmente nos exigences. Ce qui fait le succès de 1 'amour, c'est l'anxiété et non la beauté ou la laideur. C'est un amour excessif qui change le monde et un univers mental, en un monde d'une réalité "irréelle."

Parfois, rassuré pour un moment sur la fidélité d'Odette, il redevient soupçonneux de ses activités une fois de plus. Il se rappelle les expressions de son visage lorsqu'elle inventait un mensonge pour cacher un rendez-vous clandestin avec lui. Maintenant c'est lui qui éprouve toutes les terreurs de 1'amant trompé. La jalousie grandit morbidement en lui. En commençant par le drame du coucher, dans Swann, Proust établit une correspondance nécessaire entre les rapports amoureux, filiaux, amicaux ou érotiques et les tourments diaboliques de la jalousie. Le narrateur nous rappelle les pénibles instants à Combray quand il souffrait de toutes sortes d'angoisses en attendant que sa mère vienne $I^{\prime}$ embrasser et lui souhaiter une bonne nuit. Au cours d'une conversation avec Swann, Odette découvre le fait qu'il est mortellement jaloux d'elle. Un tel excès de passion chez un couple d'amants, nous fait remarquer Proust, a toujours le résultat de délier I'un des amants de I'obligation d'aimer l'autre en retour. Swann est plongé dans un désespoir complet et c'est Odette qui fuit loin de lui.

Une nouvelle attaque de désillusion en amour blesse Swann quand il reçoit une lettre anonyme qui dénonce l'immoralité d'Odette et une soi-disant passion qu'elle aurait pour Madame Verdurin. Swann fait subir à Odette un intérrogatoire à ce sujet. Mauriac a appelé cet intérrogatoire "1'enquête jalouse".2 Odette réplique aux questions anxieuses de Swann de telle sorte que Marcel la compare à un bourreau brandissant 
une hache.

L'enquête implacable de Swann peut seulement conduire à sa propre destruction. Pour n'avoir pas su trouver la perfection, l'amour ne sera pas incomplet mais déficient, sujet aux misères, aux erreurs et poussé à I'extrême, il s'achève en haine. Swann croit fortement dans la perversité d'Odette, sa corruption et sa malfaisance. Maintenant le patient devient son propre médecin et il s'impose la douleur supplémentaire de chercher comment supprimer la douleur. Swann invente de faux malentendus qu'il expose à Odette et qui ne rencontrent que l'indifférence de celle-ci. Cette indifférence persuade le malade que le malentendu imaginé était réel. Peu à peu le patient se rétablit et oublie son amour comme on se relève de maladie.

Dans un désespoir complet Swann démoralisé fréquente à nouveau les bordels parisiens. Mais comme d'autres moyens de distraction, celui-ci aussi échoue. Odette elle-même essaie de sauver Swann en le décidant à quitter Paris pour une série de voyages sur le yacht des Verdurin et elle reste loin de lui pendant plus d'un an. Au bout d'un certain temps, Swann découvre que progressivement il n'éprouve plus de désir pour Odette. Après s'être déchaîné si longtemps, le caractère maladif de cet amour s'éteint entraînant la disparition de I'amour lui-même.

La séparation physique entre Odette et Swann semble être le symbole du thème de l'aliénation spirituelle de ces deux caractères. Les thèmes de la séparation et de 1'aliénation chez Proust se répètent sans fin mais jamais de façon monotone tant leur variantes sont adr ites. Ces variations existent sous la forme d'obstacles divers. Si nous commençons au début du roman, Du Côté de chez Swann, nous voyons que Swann est reçu dans la famille de Marcel à Combray seul, sans sa femme parce qu'elle a mauvaise réputatation. C'est une barrière sociale. L'ostracisme est $\mathcal{L}^{\prime}$ une des préoccupations principales du clan Verdurin et c'est Mme Verdurin qui élève une barrière entre Swann et odette. La position sociale et 1 'amour-compétitif 
s'unissent pour former cet obstacle. A l'origine, Swann, 1'invité à dîner, empêche la mère de Marcel de venir le voir à 1 'heure du coucher, et puis Marcel sanglotant et malade sépare ses parents pour la nuit. Ayant conquis et possédé ce qu'il désirait, un homme perd 1'impulsion instinctive de posséder. Il devient indifférent et éprouve le désir d'être seul. L'indifférence qui résulte de la conquête ou de l'habitude représente un exemple type d'obstacle chez Proust. L'amour est une torture car plus nous sommes intéressés par d'autres personnes, moins nous pouvons les atteindre et les comprendre puisque leurs images ne sont que des projections de nos propres impressions. C'est un obstacle créé par des illusions. Finalement l'obstacle de la jalousie due à un amour qui n'est pas mutuel et qui est inspiré par la peur de la séparation constitue une partie intégrale de l'amour.

L'amour se compose non seulement d'une stérilité passive, mais aussi d'une phase active et destructive. Tout d'abord il semble apporter un soulagement à cette stérilité spirituelle et promettre une vie différente et plus féconde. Mais cette promesse ne se réalise pas, au contraire car sa poursuite ne laisse aucune place aux dons artistiques et cette partie de la nature de Swann est terriblement réduite. Swann doit enfin constater les ravages de 1 'amour. Proust représente cette réalité par des images qui peignent I'humiliation du corps, la dégradation des capacités mentales et morales et la destruction de l'âme. La puissance de la force destructice de l'amour est mise en évidence dans "le désir de mourir". Alors que 1'amour devrait être une délivrance de sa souffrance, ses tourments deviennent si insupportables qu'il va jusqu'à souhaiter la mort d'odette.

Chez Swann, la jalousie devient un état chronique. L'auteur le compare à "un morphinomane" ou à "un tuberculeux" (p. 307) et indique que la maladie de Swann vient d'entrer dans son stade incurable. Des images dominent, au lieu d'un langage obstrait pour exprimer cet état d'esprit. Ainsi, $1^{\prime}$ amour de Swann pour Odette devient un fait médical. Puisque la jalousie dématérialise les choses, 1'amour jaloux devient maladif. La 
présence d'Odette peut seule donner à Swann un soulagement temporaire de ses tourments, elle agit sur lui comme une drogue. Tandis que $1^{\prime}$ habitude de $1^{\prime}$ amour comme un calmant nourrit le moi en lui donnant un triomphe illusoire et imaginé sur d'innombrables rivaux.

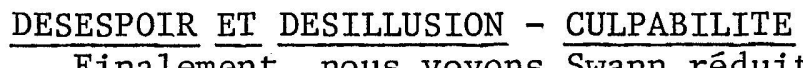

Finalement, nous voyons Swann réduit à une hystérie totale. Voilà le sommet de son amour destructeur pour Odette. Nous constatons qu'en cent soixante pages, Proust a amené, avec beaucoup de vraisemblance, Swann d'un état d'indifférence totale au plus profond désespoir. Sa souffrance mentale est si insupportable qu'il recherche avec espoir les signes de quelque maladie fatale en son corps. Les images médicales sont unies à celles d'un amour plein de culpabilité dont l'expiation est une souffrance causée par la jalousie et représentée par une douleur physique. Swann cherche la douleur physique comme une sorte de rétribution, d'expiation d'un péché passé. L'amour coupable était annoncé dès l'épisode du drame du coucher. Quand 1 'enfant réussit à avoir sa mère près de 1 ui pendant une nuit, il sent que c'est une victoire qu'il a gagnée contre elle et a l'impression qu'il la fait vieillir et mourir. Son triomphe devient alors sa propre punition. Quand le Baron de Charlus apparait tout d'abord à Balbec, ses goûts homosexuels sont comparés à des conspirateurs criminels. En association avec les rapports de l'amour coupable, on trouve le besoin de se confesser pour expier. C'est pourquoi Swann se sent obligé de parler d'Odette à chaque occasion et pourquoi le narrateur mentionne sans cesse le souvenir douloureux du drame du coucher.

REALISATION - PHASE DE REVE

Les rêves sont utilisés comme des souhaits cachés et comblés qui apportent du plaisir à l'individu. De là provient un besoin de se confesser. Ce besoin est accompagné de tortures morales et d'un désir d'expier des péchés réels et imaginaires. Cependant les rêves 
peuvent aussi révéler la réalité mieux que les moments éveillés. Presque à la fin de son amour pour Odette, les rêves de Swann lui permettent de voir objectivement sa maitresse et lui donnent un certain recul pour mieux juger la futilité complète de son désespoir.

Le tournant crucial dans le cours de l'amour de Swann se produit pendant la soirée musicale de Madame de Sainte-Euverte. En entendant de nouveau "la petite phrase de Vinteuil," il subit la révélation de l'indignité de son amour passé, il comprend la misère de son état prése $t$ comparé aux espoirs qu'il avait nourris d'un amour heureux. Il découvre aussi l'existence en lui-même de profondeurs ignorées. Jusqu'à ce moment il avait été encouragé par l'illusion que les choses pourraient s'améliorer selon ses désirs, maintenant il sait qu'Odette ne l'aimera jamais plus. Avec cet amour qui meurt et avec l'espoir d'échapper à son obsession, Swann reprend une fois de plus son étude sur Vermeer.

\section{INDIFFERENCE - ALIENATION}

Le glas final de sa passion se fait entendre lors d'un rêve que Swann fait sur Odette et les Verdurin. Dans ce rêve, il la voit objectivement pour la première fois depuis qu'il l'aime. Odette n'est plus transfigurée et ne ressemble plus à un portrait de Botticelli, provoquant la séparation entre la vie esthétique et la vie affective de Swann. Un amour naît grâce à l'imagination éveillée d'un individu. Il meurt à cause d'un rêve inconscient. Quand Swann s'éveille, c'est un homme transformé. I1 y a maintenant une porte qui s'ouvre par laquelle les dons artistiques de Swann vont pouvoir se développer. La poursuite de 1'amour était pour Swann une recherche de valeurs éternelles entreprise dans le domaine de la réalité extérieure alors que 1 'oeuvre d'art ne peut être créée que par une descent au plus profond du moi. Swann avait comparé Odette au portrait de Zéphora et cette comparaison était un effort pour la doter d'une signification plus profonde. Les tensions entre les réalités intérieures et extérieures, entre le monde de l'art et 
celui de l'amour, entre la vérité générale et individuel déterminent la structure d'Un Amour de Swann.

INDIFFERENCE - ESTHETIQUE

La femme est la cause de toutes les transgressions de 1'homme. E1le est 1'instrument de tous ses tourments. Mais 1a douleur qu'il éprouve dans un amour frustré inspire son art et lui fournit les moyens de se connaître lui-même. Proust considère que la vie d'un homme est un effort perpétuel pour pénétrer 1'inconnu, 1'esprit de la personne aimée, 1'âme de 1'ami admiré, la société dans laquelle il vit. Le désir est en vérité la condition nécessaire de 1'amour, mais ce désir de pénétrer l'inconnu dans les autres ne se satisfait jamais. Le thème qui revient sans cesse dans 1'oeuvre de Proust est celui d'une perpétuelle désillusion qui mène à un niveau plus élevé de connaissance. Plus 1 'homme se détourne d'une désillusion constante et plus clairement il conçoit que la seule réalité qu'il puisse espérer atteindre est celle de sa propre expérience. L'amour s'attache aux êtres cruels car ils comblent le désir de souffrance des amants. Dans ce cas, le concept de l'amour remplace celui du Destin. Odette, Rachel, Gilberte, Albertine et Morel portent $1 e$ virus de la maladie mais n'en sont pas atteints eux-mêmes quoi qu'ils communiquent avec des autres. Ils sont trop réalistes pour s'inquiéter de la recherche de vérités éternelles, idéales. La recherche de $1^{\prime}$ argent ou la recherche d'une position sociale est le grand motif d'action de leur monde. L'amour avec sa réalité physique crée périodiquement les conditions nécessaires à un entraînement esthétique libre, délivré de la réalité et dirigé dans la direction de la vérité éternelle.

Swann maintenant pense à Combray et à $1^{\prime}$ image de Mme de Combremer. Avec la conclusion du cycle de 1'amour de Swann, un début qui nâ̂t dans l'indifférence et dont la fin présente un retour à l'indifférence, Swann, une fois de plus, s'embarque avec un enthousiasme renouvelé dans une nouvelle aventure 
amoureuse. D'après Proust, un amour absolu n'existe pas. Aussitôt qu'un cycle s'achève, un autre recommence. Comme témoignent I'intérêt de Swann pour Mme de Cambremer et 1 'aventure amoureuse de Marcel qui va du cycle de Gilberte au cycle d'Albertine. Tant que $1^{\prime}$ homme recherchera une vérité universelle en amo, il sera entraîné dans ce tourbillon.

\section{CONCLUSION}

La nature cyclique de 1'amour proustien représente les efforts infructueux de I'homme pour atteindre une vérité éternelle. Cependant, il est impossible de saisir cette vérité éternelle, affirme l'auteur, du moins par le moyen de l'amour. Néanmoins, le cycle produit une phase de pure création esthétique. Proust affirme que cette phase représente le véritable chemin vers la vérité éternelle. Alors, nous pouvons dire que, chez Proust, le désespoir dû̀ à un amour est un élément nécessaire à la création artistique.

Proust est extrêmement prudent et restreint dans son choix quand il désigne ceux qui peuvent aimer. Seules quelques personnes ont la capacité et le désir d'aimer. Elles sont définies par Proust comme inte1ligentes, cultivées et riches. Elles possèdent la richesse qui leur permet le luxe d'aimer et l'intelligence qui leur donne la possiblilté de développer cet amour de façon intellectuelle. I1 n'admet pas l'existence de 1'amour absolu, l'illusion classiquement romantique ou même $1 \mathrm{a}$ possibilité d'un amour limité dans lequel deux individus trouvent le bonheur. L'amour donc est synonyme de souffrance et seule une séparation volontaire loin de cet amour peut apporter un certain soulagement.

I1 semble que seul celui qui a des dons artistiques possède aussi des dons correspondants pour 1 'amour au sens proustien de ce terme. L'entraînement intellectuel et affectif dans 1'appréciation des deux naît des mêmes qualités esthétiques. La différence entre les deux est que le don d'aimer opère par rapport à un autre être qui est égoĩste et n'aime pas en retour, ce qui conduit fatalement au désespoir et à la désillusion. Le don de I'art, par ailleurs, n'a pas besoin de 
réciprocité et par 1à est constant. L'amour conduit à une stérilité esthêtique, un état total et inutile d'obsession et de désespoir. L'art représente la création esthétique qui est un état de bonheur et d'accomplissement pour I'individu. Néanmoins 1 'individu se soumet lui-même à des périodes alternées de chacun d'eux dans le cycle immuable de l'amour.

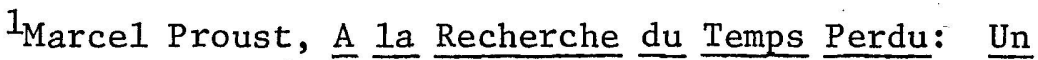
Amour de Swann (Paris: Gallimard Bibliothèque de la Pléĩade, 1960, pp. 238-239. Toutes les références dans cet essai renvoient à cette édition.

2François Mauriac, Du Côté de Chez Proust (Paris: La Table Ronde, 1947), p. 28 .

\section{Bibliographie}

\section{Livres}

Fernandez, Ramon. A la Gloire de Proust. Paris:

Editions de la Nouvelle Revue Critique, 1943. Germain, André. Les Clés de Proust. Paris: Editions Sun, 1953.

Hindus, Milton. A Reader's Guide to Marcel Proust.

New York: Farrar, Straus and Cudahy, 1962.

- The Proustian Vision. New York: Columbia

University Press, 1954 .

LaGarde, André, Laurent Michard, et al. XXe Siècle:

Les Grands Auteurs Français. Paris: Bordas, 1962.

Lindner, Gladys Dudley. Marcel Proust. California:

Stanford University Press, 1942.

Mauriac, François. Du Côté de chez Proust. Paris:

La Table Ronde, $1 \overline{947}$.

Miller, Milton L., M.D. Nostalgia; A Psychoanalytic

Study of Marce1 Proust. Boston: Houghton Mifflin Co., 1956 .

Painter, George D. Proust: The Later Years. Boston: Little, Brown and Company, 1965 .

Proust, Marcel, A la Recherche du Temps perdu, Un Amour de Swann. Paris: Editions Gallimard, Bibliothèque de la Pléiade, 1960. 
Vallée, Claude. La Féerie de Marcel Proust. Paris: Fasquelle Editeurs, 1958 .

\section{Publications Périodiques}

Be11, William S. "Proust's Un Amour de Swann: A Voyage to Cytherea," L'Esprit Créateur, V (Printemps 1965), 26-37.

Brée, Germaine. "Jean Santeuil: An Appräisal,"

L'Esprit Créateur, V (Printemps, 1965), 14-25. Hornsby, Jessie L. "Le 'Nouveau Roman' de Proust," L'Esprit Créateur, VII (Eté, 1967), 67-80. Houston John Porter. "Literature and Psychology: The Case of Proust," L'esprit Créateur, V (Printemps, 1965), 3-13.

Kolb, Philip. "Proust's Protagonist as a 'Beacon'," L'Esprit Créateur, V (Printemps, 1965), 38-47. Naughton, Helen Thomas. "A Contemporary Views Proust," L'Esprit Créateur, V (Printemps, 1965), 48-55.

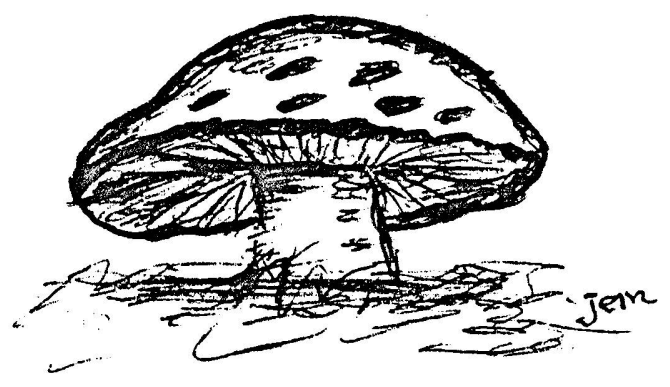


Un Diagramme de 1 'amour cyclique proustien

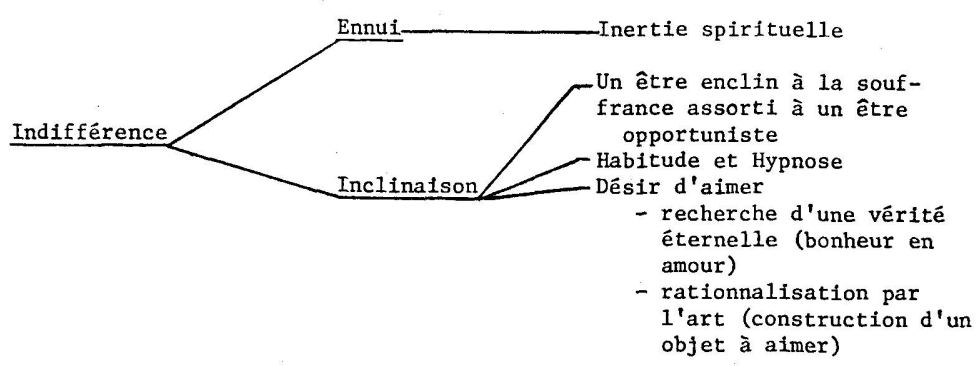

IMPULSION ACCIDENTELLE - . . . . . . . . . . . . . . .

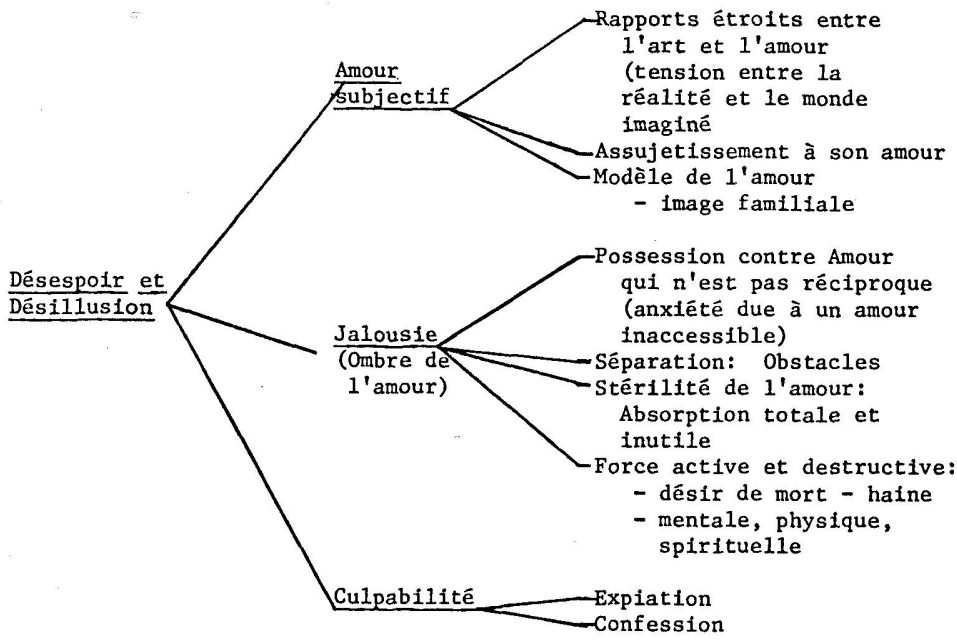

REALISATION - PHASE DE REVE - . . . . . . . . . . . . . . .

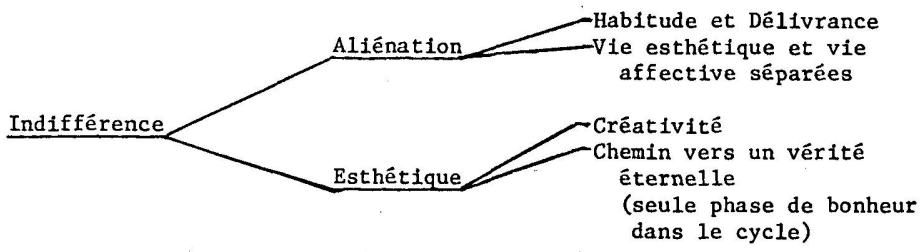

